\title{
Shprintzen-Goldberg Syndrome: A Rare Disorder
}

\author{
Attia Bari, Nadia Sadaqat, Nadia Nawaz and Iqbal Bano
}

\begin{abstract}
The Shprintzen-Goldberg syndrome (SGS) or velo-cardio-facial syndrome (VCFS) is an extremely rare disorder of connective tissue with a characteristic facial dysmorphism, marfanoid features, craniosynostosis, dolichocephaly, cardiovascular anomalies and mild to moderate mental retardation. It may be a de novo gene mutation or inherited as an autosomal dominant disorder having SKI gene and Fibrillin-1 gene (FBN1) mutations, located on chromosome 15q21.1. We report a case of a 3-month, developmentally delayed male infant admitted to the hospital with syndromic facies, craniosynostosis, joint laxity and on echocardiography, aortic root dilatation. A probable diagnosis of SGS was made on the clinical grounds. We did not have the facility for genetic chromosomal analysis. He was discharged with family counselling and follow-up for future developmental rehabilitation.
\end{abstract}

Key Words: Shprintzen-Goldberg syndrome, Velo-cardio-facial syndrome, craniosynostosis, Marfanoid features, Connective tissue disorders.

\section{INTRODUCTION}

Shprintzen-Goldberg syndrome (SGS), also known as velo-cardio facial syndrome (VCFS), is an extremely rare connective tissue disorder having multi-organ involvement. The syndrome was first documented by Shprintzen in 1982 as an isolated clinical entity. ${ }^{1}$ Since then, very few cases have been included in the literature; as worldwide, fewer than 50 cases of SGS have been reported. 2 The SGS shares many features with the Marfan syndrome and it may be misdiagnosed as later. ${ }^{3} \mathrm{~A}$ set of distinctive features are present in the affected individuals. Most common feature is pre-mature fusion of skull sutures resulting in craniosynostosis (long narrow head), Marfanoid habitus, long slender fingers and an unusual hypermobility of joints. The distinctive facial features in patients with SGS are: widely spaced eyes, downward slanting palpebral fissures, low-set ears, micrognathia and a high arched palate.2,4 Delayed developmental motor milestones, hypotonia and certain degree of intellectual disability is also common in SGS. 4,5

SGS has many similarities with Marfan syndrome and Loeys-Dietz syndrome, though SGS patients are more likely to have intellectual disability; and cardiac defects are more common in Marfan syndrome and Loeys-Dietz syndrome. Ectopia lentis, a uniform feature of Marfan syndrome, is not present in SGS and Loeys-Dietz syndrome. ${ }^{6}$ SGS mostly results from de novo gene mutations without any affected family member. SGS is caused by mutation in the SKI gene and Fibrillin-1 gene

Department of Pediatric Medicine, The Children's Hospital and Institute of Child Health, Lahore, Pakistan

Correspondence: Dr. Attia Bari, Department of Pediatric Medicine, The Children's Hospital and Institute of Child Health, Lahore, Pakistan

E-mail:drattiabari@gmail.com

Received: November 20, 2018; Accepted: February 06, 2019
(FBN1), located on 15q21.1, the same gene responsible for Marfan syndrome, resulting in similar features like Marfan syndrome. ${ }^{7}$

\section{CASE REPORT}

A 3-month boy, born from consanguineous marriage, presented with vomiting and loose stools. On examination, he was a thin lean child with marked failure to thrive. On physical examination, he was found to have Marfanoid habitus along with craniosynostosis consistent with SGS. The features which were consistent with this syndrome were: craniosynostosis (Figure 1), low set ears, prominent eyes, downward slanting palpebral fissures, small lower jaw (Figure 2), long, slender fingers (arachnodactyly) (Figure 3 ), joint hypermobility (Figure 4), high arched palate, hypotonia, pectus excavatum and flat feet (pes planus). He also had developmental delay in the form of no neck holding at 3 months of age and no social smile. His echocardiography revealed aortic root dilatation. His eye evaluation was normal with no evidence of ectopia lentis.

On the basis of clinical findings, he was diagnosed as a probable case of SGS. His active problem was managed

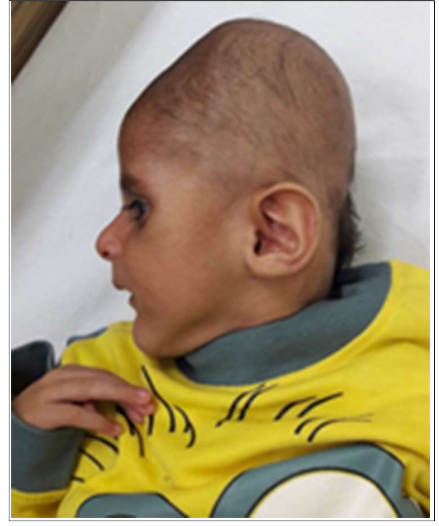

Figure 1: Craniosynostosis.

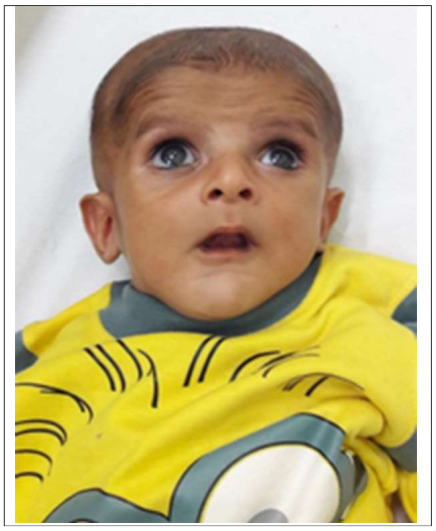

Figure 2: Low set ears and downward slanting palpebral fissures. 


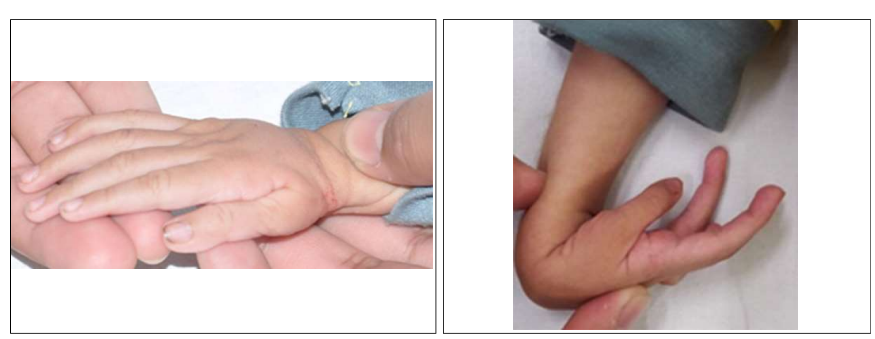

Figure 3: Long fingers.

Figure 4: Hyper-mobile joints.

and he was discharged home with future follow-up in developmental pediatric and pediatric cardiology.

\section{DISCUSSION}

SGS is a rare genetic disorder. The most common feature in SGS is craniosynostosis. The early fusion of skull sutures prevents skull growth and leads to a long narrow head and facial dysmorphism. The elongated dolichocephalic head of our patient is shown in Figure 1. This feature of our patient is consistent with various case reports from all over the world.2,5,8 The other characteristic features of SGS are hypertelorism, exophthalmos, antimongoloid slant of palpebral fissures, low-set ears, high arched palate and mandibular hypoplasia.5,8 This patient had all of these characteristics. A case report by Yalçintepe et al. from Turkey reported SGS in a 15-month male patient with dysmorphic facial appearance, dolichocephaly, prominent forehead, prominent eyes, low-set and posteriorly rotated dysplastic ears, micrognathia, high arched palate and arachnodactyly. ${ }^{4}$

Children with SGS often have a Marfanoid habitus because their body resembles those of Marfan syndrome. They may have long limbs, arachnodactyly, pectus-carinatum or pectus-excavatum, hypermobility of joints and scoliosis. The features in this patient suggestive of SGS were long fingers, hypermobile joints, pectus-carinatum and prominent eyes. Marfan syndrome is characterised by enophthalmos and, in contrast, because of the existing craniosynostosis, SGS patients have proptosis of eyes. ${ }^{3,4}$

Although SGS has typical findings, there is significant overlap of phenotype with both Marfan syndrome and Loeys-Dietz syndrome. However, SGS patients are more likely to suffer from intellectual disability, mild to moderate developmental delay and hypotonia as compared to other two conditions. This patient had developmental delay similar to the majority of affected individuals reported in various case series. ${ }^{4,5}$

The most common immediate cause of death is cardiovascular complications due to progressive aortic root dilation. Echocardiography and cardiologist consultation are necessary for early detection of these defects. To prevent the risk of rupture, the aortic root must be evaluated routinely in these children. ${ }^{7}$ In Marfan syndrome and in Loeys-Dietz syndrome, the cardiac abnormalities are more common and more severe than found in SGS. Cardiac anomalies found in Marfan syndrome are thoracic aortic aneurysms/dissections; and in Loeys-Dietz syndrome, there is generalised arterial tortuosity, aortic and peripheral arterial aneurysms/ dissection. 6 In this patient, echocardio-graphy revealed aortic root dilatation comparable with case report from India in which two-dimensional echo showed marked aortic root dilatation in a 5-year child who presented with umbilical hernia, and his features suggestive of SGS. 5

Phenotypic characteristics can be variable, and differential diagnosis must be done carefully. However, $S K I$ is the only gene which is reported as associated with SGS. A study by Doyle et al. showed that mutations to enhance TGF- $\beta$ signalling are sufficient to cause human phenotypes which are associated with low TGF- $\beta$ signalling states like craniosynostosis, altered palatogenesis and aortic aneurysm. ${ }^{9}$ The authors did not have the opportunity to perform molecular analysis of this patient.

SGS is a rare multiple congenital anomaly syndrome characterised by Marfanoid habitus, craniosynostosis, dolichocephaly, intellectual disability and dysmorphic facial features. Due to developmental delay and cardiac defects, the treatment and follow-up need lifelong multidisciplinary approach.

\section{REFERENCES}

1. Shprintzen RJ, Goldberg RB. A recurrent pattern syndrome of craniosynostosis associated with arachnodactyly and abdominal hernia. J Craniofac Genet Dev Biol 1982; 2:65-74.

2. Topouzelis N, Markovitsi E, Antoniades K. ShprintzenGoldberg syndrome: Case report. Cleft Palate Craniofac J 2003; 40:433-6.

3. Al Kaissi A, Marrakchi Z, Nassib NM, Hofstaetter J, Grill F, Gange $R$, et al. Craniosynostosis, Scheuermann's disease, and intellectual disability resembling Shprintzen-Goldberg syndrome: A report on a family over 4 generations. Medicine (Baltimore) 2017; 96:e6199.

4. Yalçintepe S, Yuregir OO, Bozdogan ST AH. ShprintzenGoldberg syndrome: Case Report. Meandros Med Dent J 2018; 19:175-7.

5. Shah B, Sahu S, Kalakoti P, Yadav S, Syed MMA, Bhattad B, et al. Shprintzen-Goldberg syndrome presenting as umbilical hernia in an Indian child. Australas Med J 2014; 7:51-7.

6. Hoffjan S. Genetic dissection of Marfan syndrome and related connective tissue disorders: An update 2012. Mol Syndr 2012; 3:47-58.

7. Yadav S RG. Shprintzen-Goldberg syndrome: A rare disorder. Pan African Med J 2016; 23:227.

8. Ingle SY, Shah P, Karande P. A rare case of Shprintzen Goldberg syndrome. J Evolution Med Dent Sci 2016; 5:914-6.

9. Doyle AJ, Doyle JJ, Bessling SL, Maragh S, Lindsay ME, Schepers D, et al. Mutations in the TGF- $\beta$ repressor SKI cause Shprintzen-Goldberg syndrome with aortic aneurysm. Nut Genet 2012; 44:1249-54.

$$
\text { .......... }
$$

